\title{
Influence of thermal conditions on the electrocaloric effect in a multilayer capacitor based on doped $\mathrm{BaTiO}_{3}$
}

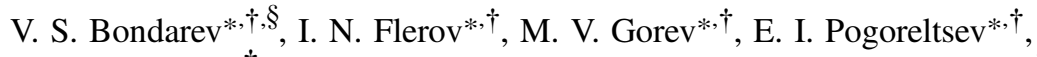 \\ M. S. Molokeev*, ${ }^{*}$, E. A. Mikhaleva*, A. V. Shabanov* and A. V. Es'kov \\ *Kirensky Institute of Physics, Federal Research Center KSC SB RAS, Krasnoyarsk 660036, Russia \\ †Siberian Federal University, Krasnoyarsk 660074, Russia \\ $\$$ International Laboratory "Materials and Structures for Electro- and \\ Magnetocaloric Energy Conversion”, ITMO University, St. Petersburg 197101, Russia \\ §bondarev@yandex.ru
}

Received 23 October 2017; Revised 3 December 2017; Accepted 4 December 2017; Published 29 December 2017

\begin{abstract}
We present the results of studies of thermal expansion, heat capacity, permittivity, polarization and the influence of different thermal conditions on the intensive electrocaloric effect (ECE), $\Delta T_{\mathrm{AD}}$, in a commercial multilayer capacitor based on doped $\mathrm{BaTiO}_{3}$. Investigations in a wide temperature range revealed one anomaly in the behavior of the physical properties at about $290 \mathrm{~K}$ characteristic for the relaxors. Direct measurements showed a high reversibility of ECE under equilibrium thermal conditions. Good agreement was found between the values of $\Delta T_{\mathrm{AD}}$ determined by direct and indirect measurements at the electric field up to $15.4 \mathrm{kV} / \mathrm{cm}$. Quasi-isothermal conditions lead, firstly, to decrease of large $\Delta T_{\mathrm{AD}} \approx 0.94 \mathrm{~K}$ obtained in adiabatic conditions under $E=308 \mathrm{kV} / \mathrm{cm}$ to $0.87 \mathrm{~K}$, and, secondly, the appearance of a difference between the values of $\Delta T^{\mathrm{ON}}$ and $\Delta T^{\mathrm{OFF}}$ determined when the electric field is applied and removed. Using this phenomenon and changing the frequency of the periodic electric field, $E=15.4 \mathrm{kV} / \mathrm{cm}$, the effect of cooling was obtained equal to $\left[\left(\Delta T^{\mathrm{ON}}+\Delta T^{\mathrm{OFF}}\right) / 2\right]_{\max }=-0.032 \mathrm{~K}$. The results obtained are useful for further development of the electrocaloric refrigeration technique without thermal switches.
\end{abstract}

Keywords: Electrocaloric effect; ferroelectrics; layered structures; nonequilibrium conditions; phase transitions.

\section{Introduction}

In recent years, considerable attention has been given to solids showing large caloric effects (CE) due to their potentiality to be used as effective solid refrigerants in cooling devices for various aims. ${ }^{1-5}$ Among caloric phenomena of different physical origins, electro (ECE)-, magneto (MCE)and baro (BCE)-caloric effects, ECE is the most simple in a realization. Indeed, in such a case, there is no necessity in external attributes such as magnets or installations producing pressure. To produce an electric field, it is enough to apply the voltage to electrodes on a surface of the electrocaloric (EC) element.

Thermodynamic equations describing the reversible change in entropy, $\Delta S_{\mathrm{ECE}}$, at $T=$ const (extensive ECE), and temperature, $\Delta T_{\mathrm{AD}}$, at $S=$ const (intensive $\mathrm{ECE}$ ), under electric field variation

$$
\Delta S_{\mathrm{ECE}}=\int(\partial P / \partial T)_{E} d E, \quad \Delta T_{\mathrm{AD}}=-\left(T / C_{p}\right) \Delta S_{\mathrm{ECE}}
$$

show that both values depend not only on electric field strength $E$ but also on the rate of a change in the polarization with temperature. Thus, the most preferable temperature region to obtain large values of ECE is near the ferroelectric phase transition point where the $(\partial P / \partial T)_{E}$ derivative reaches the maximum.

The simplicity of ECE realization is one of the main reasons of the active investigations of caloric efficiency of ferroelectrics at the present time. Moreover, the search of ways of caloric efficiency increase continues despite of a serious disadvantage of these materials associated with rather high conductivity leading to a release of Joule heat under applying electric field. ${ }^{1,6}$

Large values of ECE for different bulk ferroelectric systems comparable to $\mathrm{MCE}$ and $\mathrm{BCE}^{3-5}$ were obtained only in theoretical calculations which were usually performed under the electric field $E$ of very high strength. For example, $\Delta T_{\mathrm{AD}} \approx 12 \mathrm{~K}$ for the ceramic $\mathrm{Ba}_{0.5} \mathrm{Sr}_{0.5} \mathrm{TiO}_{3}$ was predicted ${ }^{7}$ at $E=800 \mathrm{kV} / \mathrm{cm}$. However, a strong experimental increase in electric field applying to the bulk EC element is limited by rather low breakdown field for ferroelectrics which was found in Ref. 8 equals to $24 \mathrm{kV} / \mathrm{cm}\left(\Delta T_{\mathrm{AD}} \approx 1 \mathrm{~K}\right)$ for $\mathrm{Ba}_{0.73} \mathrm{Sr}_{0.27} \mathrm{TiO}_{3}$. Nevertheless, a serious success was reached in the way of improving the dielectric strength of ceramics of

$\S_{\text {Corresponding author. }}$

This is an Open Access article published by World Scientific Publishing Company. It is distributed under the terms of the Creative Commons Attribution 4.0 (CC-BY) License. Further distribution of this work is permitted, provided the original work is properly cited. 
$\mathrm{BaTiO}_{3} \cdot{ }^{9}$ It was shown that the decrease in the grain size from $41 \mu \mathrm{m}$ to $1.3 \mu \mathrm{m}$ and increase in density are accompanied by increase in the breakdown field from $90 \mathrm{kV} / \mathrm{cm}$ to $180 \mathrm{kV} / \mathrm{cm}$. One can think that a corresponding increase in the density of ceramic samples will lead to a decrease in the conductivity. Another way to avoid breakdown and increase at least the intensive ECE is associated with using thin ferroelectric films. In such a case, rather low voltage is enough to realize high electric field and as a result large value of $\Delta T_{\mathrm{AD}} \approx 14 \mathrm{~K}$ due to small thickness of ECEelement. ${ }^{10}$ To increase a low extensive ECE in the thin films due to their small thermal mass, layered ferroelectric systems were suggested in the form of multilayer ceramic capacitor. $^{11,12}$

A lot of papers were devoted to studies of ECE under the equilibrium thermodynamic conditions, the results of which were summarized in several reviews. ${ }^{1,2}$ Single crystals, solid solutions, ceramics, thin films and layered structures were presented as materials to be used as EC elements. A special attention was devoted to the problem of thermal switches which is common for the refrigerators with unmovable working body operating on any caloric effects. These switches providing in turn the supply and removal of heat to/ from the refrigerant are necessary to design a closed refrigeration cycle. Mechanical systems, liquid crystals, carbon nanotubes and others were suggested as thermal switches. ${ }^{11,13,14}$ Alternative decision of a problem above was suggested in the form of a cooling line without thermal switches. ${ }^{15-17}$ The idea was to generate thermal gradient in the bulk linear EC element keeping its bottom at $T_{\text {bot }}=$ const during cycling, a periodic electric field. In such nonequilibrium conditions, the temperature of the thermally isolated top of the EC element, $T_{\text {top }}$, will fluctuate and its average value will decrease down to $T_{\text {top }}<T_{\text {bot }}$. Thus, a constant thermal gradient will appear in the EC element when electric field is in turn applied and removed.

Recently, two different ferroelectric systems, a multilayer capacitor based on doped $\mathrm{BaTiO}_{3}$ (MLCBT) ${ }^{11,12}$ and a bulk crystal of triglycine sulphate (TGS), ${ }^{18,19}$ were chosen for the experimental examination of ECE in nonequilibrium thermal conditions. A heating of the $\mathrm{BaTiO}_{3}$ capacitor under periodic electric field was observed since increase in the temperature at applying $E,\left|\Delta T_{\mathrm{AD}}^{\mathrm{ON}}\right|$, exceeded this value at removal of $E,\left|\Delta T_{\mathrm{AD}}^{\mathrm{OFF}}\right| \cdot{ }^{11}$ At least two reasons can be proposed to explain these rather unexpected results related to the observed ratio $\left|\Delta T_{\mathrm{AD}}^{\mathrm{ON}}\right|>\left|\Delta T_{\mathrm{AD}}^{\mathrm{OF}}\right|$. Firstly, high strength of electric field, $E=300 \mathrm{kV} / \mathrm{cm}$, used in experiments inevitably can lead to the release of Joule heat. Secondly, the rates of switching on and off the electric field were different, $(d E / d t)_{\mathrm{OFF}}<(d E / d t)_{\mathrm{ON}}$, since to avoid the fear of destroying the capacitor during the removal of high voltage, the discharge process was performed through additional resistor. These circumstances were taken into account when studying the influence of nonequilibrium thermal conditions on intensive ECE in TGS. ${ }^{18,19}$
Measurements were performed under a low electric field, $E=2.8 \mathrm{kV} / \mathrm{cm}$, and at equal rates of its switching on and off. As a result, a decrease in the temperature $T_{\text {top }}$ compared to $T_{\text {bot }}$ of the TGS element was observed, as predicted earlier. $^{15,16}$ The temperature difference was small, $T_{\text {top }}-T_{\text {bot }}=-0.012 \mathrm{~K}$, but the main thing was that the cooling effect was observed.

This paper is devoted to precise indirect and direct measurements of ECE in MLCBT under equilibrium and nonequilibrium thermal conditions. Complex investigations of some physical properties of the multilayer capacitor (structure, permittivity, hysteresis loops, heat capacity and thermal expansion) were also performed in order to characterize the sample under study and to determine the optimal parameters for the study of ECE.

\section{Experimental}

A commercially available MLCBT $(100 \mu \mathrm{F})$ consisting of 200 interdigitated layers of the doped $\mathrm{BaTiO}_{3}$ and $\mathrm{Ni}$ electrodes was used (Fig. 1(a)). The structural morphology of the cut perpendicular to the ferroelectric and metal layers was examined using a scanning electron microscope (SEM) Hitachi TM3000 (Hitachi High - Technologies Co., Ltd., Tokyo, Japan). The results presented in Figs. 1(b) and 1(c) clearly show that alternating layers of $\mathrm{BaTiO}_{3}$ (light broad stripes) and $\mathrm{Ni}$ (dark narrow stripes) components are regular and in close contact with each other. The thickness of the electrodes and ferroelectric layers is 2 and $6.5 \mu \mathrm{m}$, respectively. The $\mathrm{BaTiO}_{3}$ layers consist of relatively densely packed grains with the average size varied from 1 to $3 \mu \mathrm{m}$ (Fig. 1(c)). Damage to the grains such as cracking is not observed.

To avoid poling and hysteresis loss leading to a deterioration in the functional properties of the capacitor, the $10 \mathrm{~V}$ maximum voltage (i.e., $\sim 15 \mathrm{kV} / \mathrm{cm}$ ) was recommended by the manufacturer. Due to rather thin dielectric layers, this magnitude is well below the breakdown field for MLCBT, since according to the related measurements of ECE, ${ }^{12,20}$ the multilayer capacitor can withstand electric field above $300 \mathrm{kV} / \mathrm{cm}$.

Structural characterization of the dielectric component in MLCBT was carried out at room temperature using a PANalytical X'Pert PRO diffractometer equipped with a PIXcel solid state detector and a secondary graphite monochromator $\left(\mathrm{Cu}-\mathrm{K} \alpha\right.$ radiation). The step size of $2 \theta$ was $0.02^{\circ}$. Rietveld refinement was performed using TOPAS 4.2. ${ }^{21}$

Measurements of thermal expansion were performed using a push-rod dilatometer (NETZSCH model DIL-402C) with a fused silica sample holder. Experiments were carried out in the temperature range of $100-350 \mathrm{~K}$ with a heating rate of $3 \mathrm{~K} / \mathrm{min}$ in a dry He flux. The results were calibrated by taking quartz as the standard reference, to remove the influence of system's thermal expansion. The uncertainty in the thermal expansion measurements was about $5 \%$. 


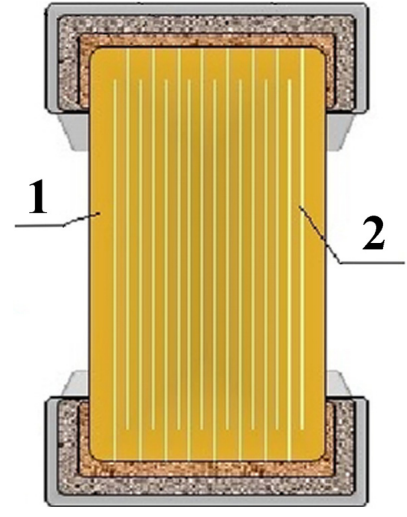

(a)

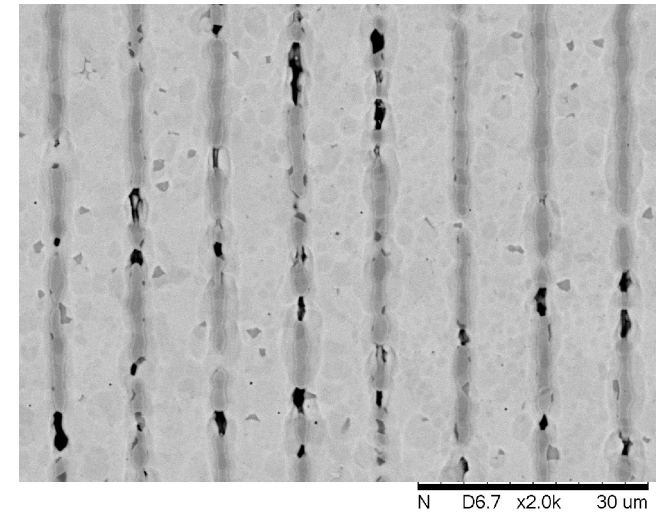

(b)

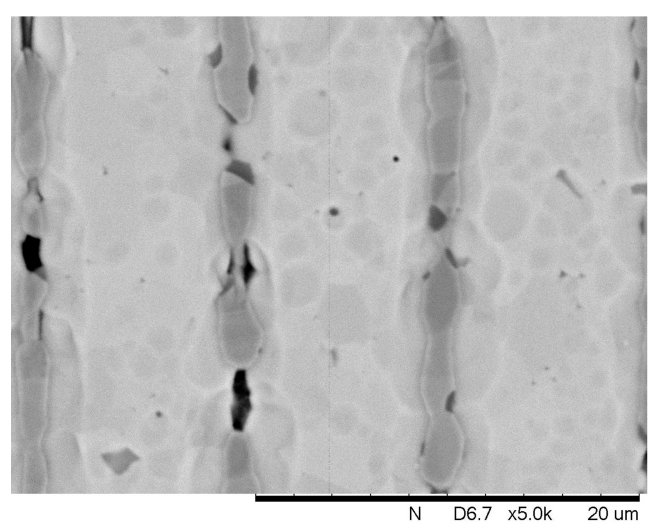

(c)

Fig. 1. The structural morphology of the multilayer condenser; (a) structure of commercial ceramic chip capacitors; 1 - ceramic dielectric; 2 - internal electrode; (b) and (c) — scanning electron microscopy images of $\mathrm{BaTiO}_{3}$ capacitor at different magnifications.

Because of the very high sensitivity to small changes in the temperature and the enthalpy/entropy, a homemade adiabatic calorimeter with three screens ${ }^{22}$ was used to perform complex studies of heat capacity, permittivity, dielectric hysteresis, pyroelectric current and ECE in equilibrium thermal conditions. MLCBT placed in the heater, consisting of a polished aluminum foil with Surface Mounted Device resistors located to its inner surface, was suspended inside the first thermal screen on thin threads ( $d \leq 0.1 \mathrm{~mm}$ ) (Fig. 2(a)). The temperature of the sample+heater system $(1,2,3)$ was monitored with a high-precision platinum resistance thermometer (5) attached to the adiabatic screen (4). The longterm stability of this thermometer is rather high $\sim 0.002 \mathrm{~K}$ and the absolute accuracy of temperature measurements was $\pm 0.01 \mathrm{~K}$. The temperature difference between the thermometer and sample was controlled and tuned using a copperconstantan thermocouple battery $\Delta T_{\text {reg }}$ whose output was supplied to the automatic control circuit connected to adiabatic screen heater (6). Measurements were performed at a pressure of about $10^{-5} \mathrm{~mm} \mathrm{Hg}$.

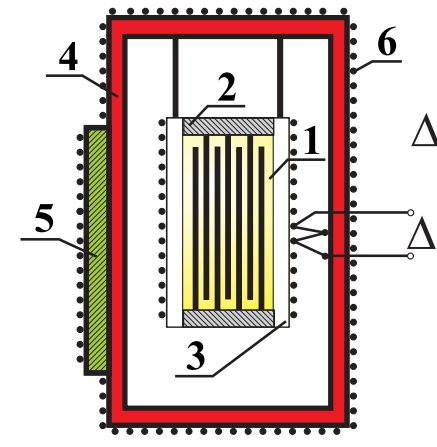

(a)

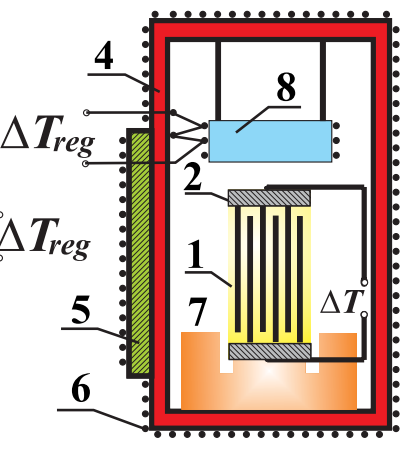

(b)
Fig. 2. Schematic of sample mounting for studying (a) the specific heat and ECE under adiabatic conditions and (b) the effect of quasiisothermal processes of switching on/off the DC and periodic electric fields.
The heat capacity of the "sample+heater+contact grease" system was measured in a wide temperature range of $120-360 \mathrm{~K}$ using discrete as well as continuous heating. In the former case, the calorimetric step was varied from 1.5 to $3.0 \mathrm{~K}$. In the latter case, the system was heated at rates of $d T / d t \approx 0.2-0.4 \mathrm{~K} / \mathrm{min}$. The heat capacities of the heater and contact grease were determined in individual experiments. The inaccuracy in the heat capacity determination did not exceed $0.2-1.0 \%$.

The temperature behavior of the permittivity $\varepsilon(T)$ was investigated using an E7-20 immittance meter at frequencies from $50 \mathrm{~Hz}$ up to $5 \mathrm{kHz}$ while heating at a rate of about $0.5 \mathrm{~K} / \mathrm{min}$ in the temperature range of $120-360 \mathrm{~K}$. The dielectric hysteresis $(P-E$ loops) was/were examined using an aixACCT EASY CHECK 300 technique. The driving-field profile was a triangular wave of amplitude $E_{\max }=18 \mathrm{kV} / \mathrm{cm}$. The frequency of the measuring electric field was $1 \mathrm{~Hz}$.

To determine the intensive ECE in MLCBT under equilibrium conditions the indirect and direct measurements were carried out. In the former case, the value $\Delta T_{\mathrm{AD}}$ was evaluated in accordance with Eq. (1) using the experimental temperature dependences of the spontaneous polarization under the different electric fields.

The procedure of direct measurements of the intensive ECE was similar to that used earlier by us studying ECE and MCE in $\mathrm{TGS}^{19}$ and $\mathrm{La}_{0.7} \mathrm{~Pb}_{0.3} \mathrm{MnO}_{3},{ }^{23}$ respectively.

The study of ECE in MLCBT under nonequilibrium thermal conditions was performed according to the scheme used previously ${ }^{19}$ (Fig. 2(b)). MLCBT (1) was placed in a copper holder (7) mounted on the bottom of the screen (4). A copper disk (8) with $d=7.94 \mathrm{~mm}$ and $h=1.89 \mathrm{~mm}$ packed in the polished aluminum foil with a heater and serving as an additional heat screen was hung over the sample to the screen cover. To ensure reliable thermal contact between the elements of the system, the vacuum grease was used. The automated control system retained the required temperature difference between the copper disk and adiabatic screen with 
an accuracy of not worse than $10^{-4} \mathrm{~K}$. The temperature of the adiabatic screen as well as base of MLCBT was kept constant. The temperature difference $T_{\text {top }}-T_{\text {bot }}$ between the "free" top and the thermostatically controlled bottom of MLCBT which occurred due to the ECE at the switching on/off electric field $E$ was detected by the copper-constantan thermocouple. The sensitivity of the temperature sensors and measurements stability were $10^{-4}$ and $5 \times 10^{-5} \mathrm{~K}$, respectively.

\section{Results and Discussion}

\subsection{Structure and physical properties}

The results of the X-ray studies are shown in Fig. 3. Rietveld refinement shows the presence of $\mathrm{Ni}$ and $\mathrm{BaTiO}_{3}$ doped with $\mathrm{Sn}^{4+}$ which gives the following chemical formula: $\mathrm{BaTi}_{0.86} \mathrm{Sn}_{0.14} \mathrm{O}_{3}$ (BTSO). Refinement was stable and gives low $R$-factors for BTSO $\left(R_{B}=1.48 \%\right)$ and Ni $\left(R_{B}=2.06 \%\right)$.

Measurements of the thermal expansion coefficient $\alpha$ were performed in three different directions of MLCBT: along (2)

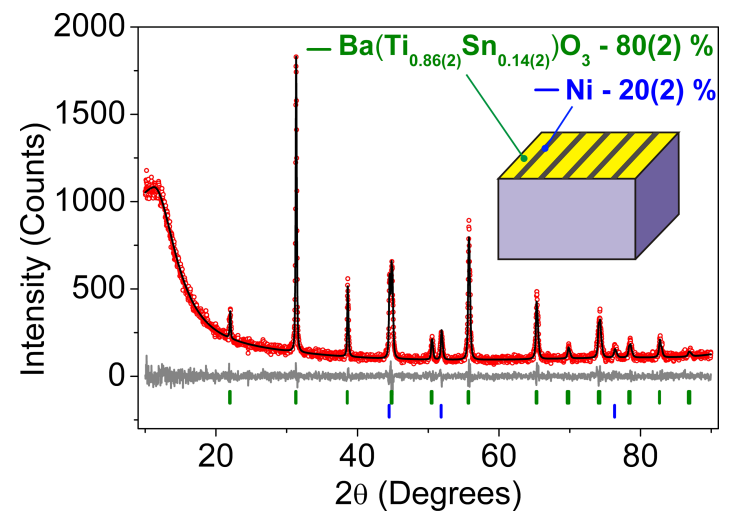

Fig. 3. (Color online) Difference Rietveld plot of $\mathrm{Ba}\left(\mathrm{Ti}_{0.86} \mathrm{Sn}_{0.14}\right) \mathrm{O}_{3}$ (green stick) with $\mathrm{Ni}$ plates (blue stick). Inset shows structure of multilayer capacitor.

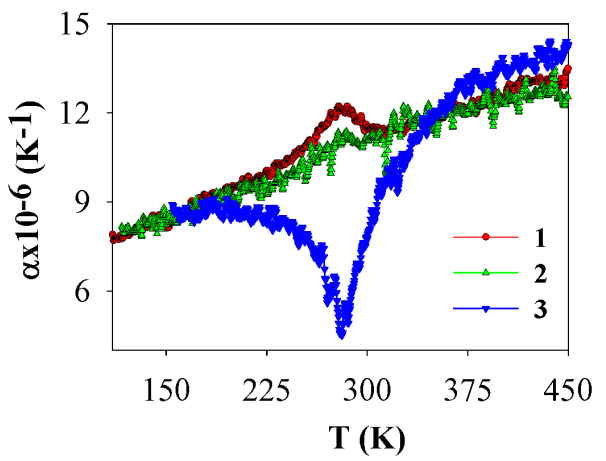

(a) and perpendicular (1) to the ferroelectric layers. One anomaly was found at about $290 \mathrm{~K}$ smeared in a wide temperature range (Fig. 4(a)). Rather strong anisotropy observed in the $\alpha(T)$ behavior can be related to the preferred orientation of the grains of the ferroelectric component during the manufacturing processes of composite consisting of two dissimilar materials, BTSO and $\mathrm{Ni}^{24-26}$

Heat capacity of MLCBT has also shown one anomaly with the maximum at $T=290 \pm 10 \mathrm{~K}$. The excess heat capacity $\Delta C_{p}(T)$ associated with the phase transition was also observed in the wide temperature range of about 200-360 K (Fig. 4(b)) as it was found for the dependence of $\alpha(T)$ (Fig. 4(a)).

The temperature dependences of the permittivity $\varepsilon(T)$ at different frequencies show a wide bump with the maximum temperature shifted with increasing frequency from $277 \mathrm{~K}$ $(f=50 \mathrm{~Hz})$ to $304 \mathrm{~K}(5 \mathrm{KHz})$ (Fig. 5(a)). It is also seen that the increase in $f$ is accompanied by a decrease in the maximum of $\varepsilon$. At the same time, the curves of $\varepsilon(T)$ for different $f$ coincide with each other above the maximum temperature. Such a behavior of permittivity of $\mathrm{BaTi}_{0.86} \mathrm{Sn}_{0.14} \mathrm{O}_{3}$ is characteristic of relaxor properties which were found for the solid solutions $\mathrm{BaTi}_{1-x} \mathrm{Sn}_{x} \mathrm{O}_{3}$ at $x>0.12 .^{27}$

Information on spontaneous polarization $P$ at various temperatures was obtained from measurements of the hysteresis loops (Fig. 6). At about $373 \mathrm{~K}$, the $P(E)$ dependence is close to linear, which is typical for paraelectrics or linear dielectrics. The presence of polarization above room temperature may be due to the polar nanoregions found in the $\mathrm{BaTi}_{1-x} \mathrm{Sn}_{x} \mathrm{O}_{3}$ solid solutions ${ }^{27}$ with $x>0.12$ which are also the cause of a significant frequency dispersion of permittivity observed by us (Fig. 5). The saturated value of $P$ equals to $\sim 18 \mu \mathrm{C} / \mathrm{cm}^{2}$ is close to polarization observed for barium titanate $\left(\sim 20-25 \mu \mathrm{C} / \mathrm{cm}^{2}\right){ }^{28}$

The investigations of $\mathrm{BaTi}_{1-x} \mathrm{Sn}_{x} \mathrm{O}_{3}$ solid solutions ${ }^{27,29,30}$ have shown that increase in $\mathrm{Sn}$ concentration is accompanied by a shift of decrease of the phase transition temperature between cubic and tetragonal phase, whereas the other two transitions are shifted to higher temperatures. Thereby, in

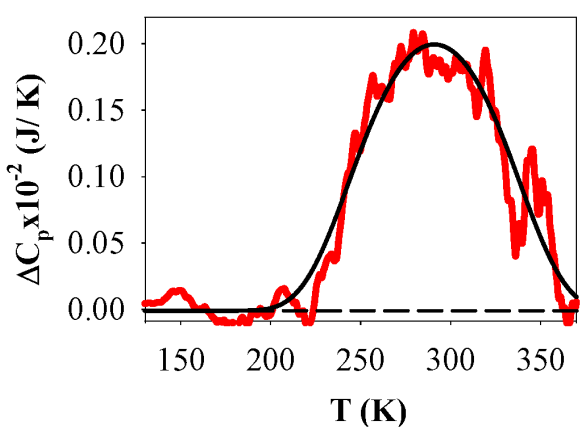

(b)

Fig. 4. (a) Thermal expansion of the multilayer capacitor in three directions: 1,2 - along the layers, 3 - perpendicular to the layers. (b) Anomalous component of the heat capacity of the multilayer capacitor. 


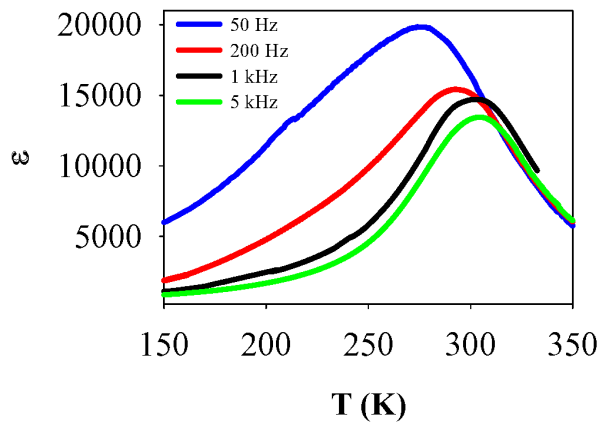

Fig. 5. Temperature dependences of permittivity at various frequencies.

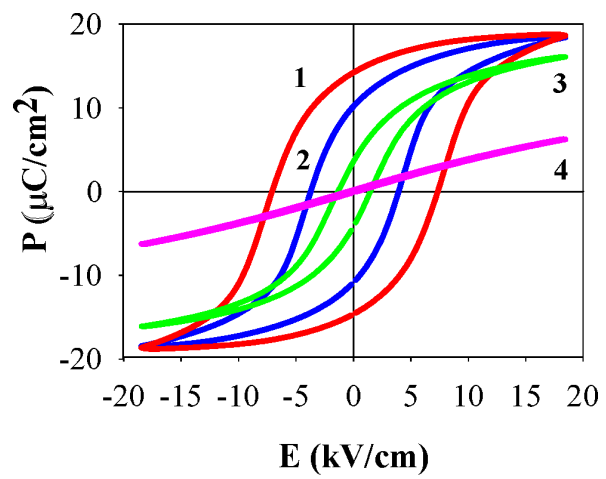

Fig. 6. Hysteresis loops at different temperatures at $99 \mathrm{~K}(1), 149 \mathrm{~K}$ (2), $260 \mathrm{~K}(3)$ and $373 \mathrm{~K}(4)$.

$\mathrm{BaTi}_{1-x} \mathrm{Sn}_{x} \mathrm{O}_{3}$ at $x>0.12$, only direct rhombohedral — cubic transition, which is often named as pinched phase transition in literature. ${ }^{27,31,32}$ Investigations of the thermal and dielectric properties (thermal expansion, heat capacity, and permittivity) confirmed the existing of one phase transition near room temperature.

\subsection{Electrocaloric effect}

An indirect determination of ECE was carried out by analyzing the field dependences of the spontaneous polarization (Fig. 7(a)). To calculate ECE in accordance with Eq. (1), the upper drop-down part of the hysteresis loop was used since the domain structure does not change significantly as the electric field decreases. The temperature dependences of the calculated intensive ECE, $\Delta T_{\mathrm{AD}}^{\mathrm{calc}}$, at different electric fields are shown in Fig. 7(b). Even under low field, the maximum of the $\Delta T_{\mathrm{AD}}^{\mathrm{calc}}$ value is observed at temperature $T_{\max }=307 \mathrm{~K}$ which is higher than the temperature of the maxima of heat capacity and thermal dilatation $(\sim 290 \mathrm{~K})$ (Fig. 4). The maximum of the $\Delta T_{\mathrm{AD}}^{\mathrm{calc}}(T)$ curves is shifted with increase in the electric field with the rate $\Delta T_{\max } / \Delta E=0.65 \mathrm{~K} /(\mathrm{kV} / \mathrm{cm})$.

Direct measurements of EC response of the MLCBT + heater system, $\Delta T_{\text {exp }}$, were carried out at adiabatic condition (Fig. 2(a)) under electric fields varied in a wide range of the strength: $0-307 \mathrm{kV} / \mathrm{cm}$. The sample + heater system was

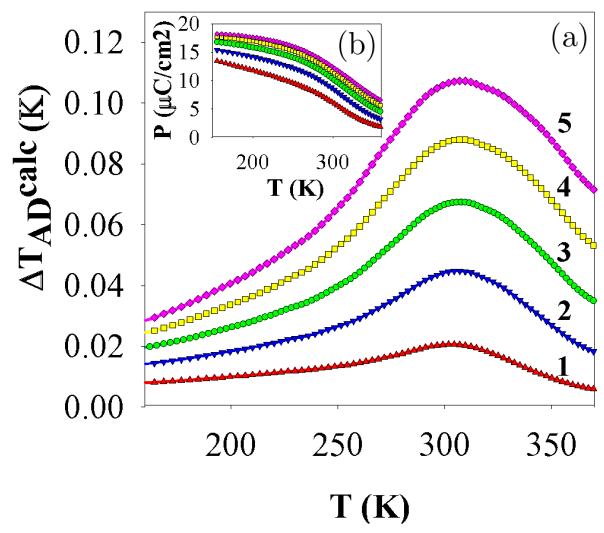

Fig. 7. (a) The temperature dependences of polarization taken from the $P-E$ loops/ (b) Calculated ECE in MLCBT versus temperature at different electric fields: $3.1 \mathrm{kV} / \mathrm{cm}(1), 6.2 \mathrm{kV} / \mathrm{cm}(2), 9.2 \mathrm{kV} / \mathrm{cm}$ (3), $12.3 \mathrm{kV} / \mathrm{cm}$ (4) and $15.4 \mathrm{kV} / \mathrm{cm}(5)$.

heated/cooled to some initial temperature in a wide temperature region from $100 \mathrm{~K}$ to $370 \mathrm{~K}$. The linear temperature drift with an optimum rate within $d T / d t \approx \pm(1-5) \times 10^{-4}$ $\mathrm{K} / \mathrm{min}$ was chosen. The switching on of electric field $\mathrm{E}$ was accompanied by abrupt increase in temperature $\Delta T_{\mathrm{EXP}}^{\mathrm{ON}}$ of the MLCBT+heater system due to ECE in the BTSO component (Fig. 8(a)). Then, the temperature drift was controlled within 5-10 min to compare it with that observed before applying the field. The electric field shutdown led to a temperature decrease $\Delta T_{\mathrm{EXP}}^{\mathrm{OFF}}$. Such a procedure was repeated at the same temperature under different electric fields. The inaccuracy in determination of the $\Delta T_{\mathrm{EXP}}$ values did not exceed $\pm 2 \times 10^{-4} \mathrm{~K}$.

It was also observed that in the process of $E=$ const, the rate of the temperature change, $d T / d t$, increases compared to that in the process of $E=0$ and decreases to the initial value just the field is switched off. Such a behavior of the temperature is associated with the release of Joule heat in the BTSO component. A relatively low electric field, $E=15.4 \mathrm{kV} / \mathrm{cm}$, applied during 5 min leads to additional increase $(\sim 10 \%)$ in the temperature change of the MLCBT + heater system. In spite of this fact, a perfect agreement was observed between the values of $\Delta T_{\mathrm{EXP}}^{\mathrm{ON}}$ and $\Delta T_{\mathrm{EXP}}^{\mathrm{OFF}}$. Information on the intensive ECE in MLCBT was obtained using the following relation between heat capacities of heater and MLCBT: $\Delta T_{\mathrm{AD}}^{\mathrm{MLCBT}}=\left(1+C_{\text {heater }} / C_{\mathrm{MLCBT}}\right)=2.5 \Delta T_{\mathrm{EXP}}$.

Very good agreement was found between the data obtained in direct (Fig. 8) and indirect (Fig. 7) measurements of $\Delta T_{\mathrm{AD}}$ at $E=15.4 \mathrm{kV} / \mathrm{cm}: \Delta T_{\mathrm{AD}}^{\mathrm{MAX}}=0.104 \mathrm{~K}$ and $\left(\Delta T_{\mathrm{AD}}^{\text {calc }}\right)^{\mathrm{MAX}}=0.107 \mathrm{~K}$. The maximum value of $\mathrm{ECE}$, $\Delta T_{\mathrm{AD}}=0.94 \mathrm{~K}$, observed in experiments under the electric field $E=308 \mathrm{kV} / \mathrm{cm}$ is higher compared to the value $\Delta T_{\mathrm{AD}}=0.55 \mathrm{~K}$ found for related capacitor at the same $E .^{12}$ Such a rather large difference between the values of $\Delta T$ is associated with additional thermal losses due to the absence of adiabatic conditions. ${ }^{12}$ 


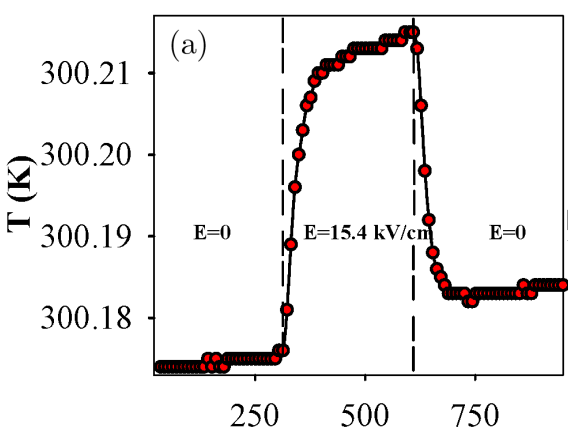

t (s)

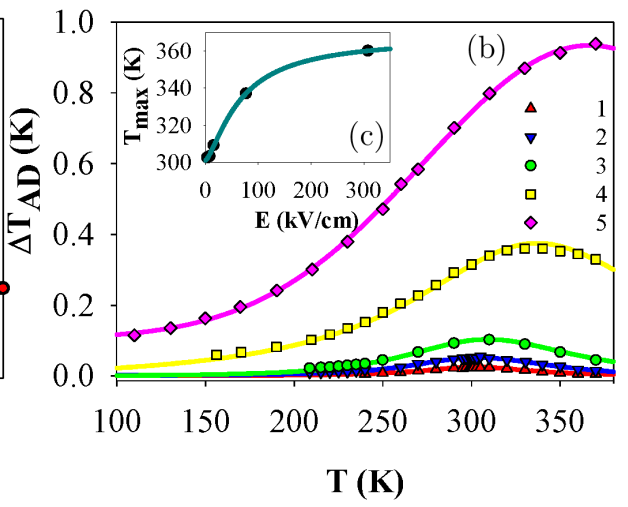

$\mathbf{T}(\mathrm{K})$

Fig. 8. (a) The time dependence of the temperature of the MLCBT + heater system under successive electric field variation at $S=$ const: $E=0 \rightarrow E=15.4 \mathrm{kV} / \mathrm{cm} \rightarrow E=0$. (b) Temperature dependence of the intensive ECE recalculated for MLCBT in the equilibrium conditions at $E=3.1 \mathrm{kV} / \mathrm{cm}(1), 7.7 \mathrm{kV} / \mathrm{cm}(2), 15.4 \mathrm{kV} / \mathrm{cm}(3), 76.7 \mathrm{kV} / \mathrm{cm}(4), 308 \mathrm{kV} / \mathrm{cm}$ (5) and (c) Dependence of $T_{\max }$ versus applied $E$.

Despite the fact that the value $\Delta T_{\mathrm{AD}}^{\mathrm{MAX}}$ was found at rather high temperature $T_{\max }=361 \mathrm{~K}$, Fig. 8(b) shows a decrease in the rate $\Delta T_{\max } / \Delta E$ with increase in $E$. Nevertheless, it is obvious that both values $T_{\max }$ and $\Delta T_{\mathrm{AD}}^{\mathrm{MAX}}$ did not reach a saturation even at such a high electric field.

Measurements of electrocaloric response in MLCBT in nonequilibrium thermal conditions were performed in accordance with scheme presented in Fig. 2(b). Such conditions can be regarded as quasi-isothermal, since only the temperature $T_{\text {bot }}$ was kept constant. At the first stage, we applied a measurement mode similar to that used earlier in Ref. 11. Since the studied MLCBT has a significant capacitance, in order to avoid the destruction of the capacitor when the electric field is removed, the discharge process was carried out through a resistance of $910 \mathrm{Ohm}$.

Fast (adiabatic) switching on/off an electric field at $T=300 \mathrm{~K}$ which is slightly below $T_{\max }$ is accompanied by a rapid increase/decrease in the temperature of the "free" top of MLCBT by a value of $\Delta T_{\mathrm{EXP}}^{\mathrm{ON}} / \Delta T_{\mathrm{EXP}}^{\mathrm{OFF}}$ due to ECE (Fig. 9). Then, at $E=$ const, the temperature relaxes slowly to the temperature $T_{\text {bot }}$, i.e., $\Delta T_{\mathrm{EXP}} \rightarrow 0$. The temperature gradient occurring on the sample led to the heat exchange between the thermostated bottom and thermodynamically "free" top of the EC element.

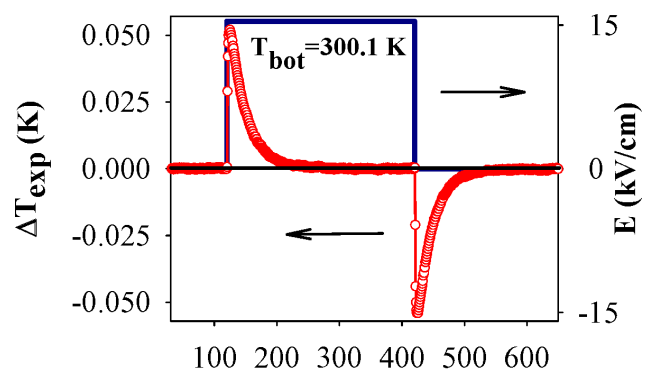

$\mathbf{t}(\mathbf{s})$

Fig. 9. The profile of electric field variation $E(t)$ and associated intensive EC response, $\Delta T_{\exp }(t)=T_{\text {top }}-T_{\text {bot }}$, at $T_{\text {bot }}=300.1 \mathrm{~K}$.
As an example, the results of measurements of ECE under $E=308 \mathrm{kV} / \mathrm{cm}$ are shown in Fig. 10. The temperature dependence of the absolute magnitude of temperature change $|\Delta T|$ obtained in the regimes of applying and removal $E$ and recalculated for the MLCBT shows that the intensive ECE is quantitatively irreversible: The value of $|\Delta T|^{\mathrm{ON}}$ is greater than $|\Delta T|^{\mathrm{OFF}}$ in a wide temperature range of $290-370 \mathrm{~K}$ similar to data observed in Refs. 11 and 12.

The maximum EC response $|\Delta T|^{\mathrm{OFF}}$ under quasiisothermal conditions is lower compared to $\Delta T_{\mathrm{AD}}^{\max }$ observed in measurements under equilibrium conditions (Fig. 8(b)). This can be due to the nonadiabatic regime of measurements and the occurrence of a heat flux from top to bottom of the EC element during the process $(E=0) \rightarrow(E \neq 0)$.

Taking into account our experience of measuring ECE in TGS in nonequilibrium thermal conditions, ${ }^{18,19}$ the measurements at the second stage were performed at the same rates of switching on and off the electric field, $(d E / d t)^{\mathrm{OFF}}=$ $(d E / d t)^{\mathrm{ON}}$. For this aim, MLCBT was also charged via a resistor of $910 \mathrm{Ohm}$.

Figure 11 shows the behavior of $|\Delta T|^{\text {OFF }}$ and $|\Delta T|^{\text {ON }}$ under $E=15.4 \mathrm{kV} / \mathrm{cm}$. It is seen that in the temperature range of $290-370 \mathrm{~K} \Delta T^{\mathrm{ON}}<\Delta T^{\mathrm{OFF}}$. The maximum difference $|\Delta T|^{\mathrm{OFF}}-|\Delta T|^{\mathrm{ON}}$ at $T=320 \mathrm{~K}$ is $\sim 0.005 \mathrm{~K}$ at

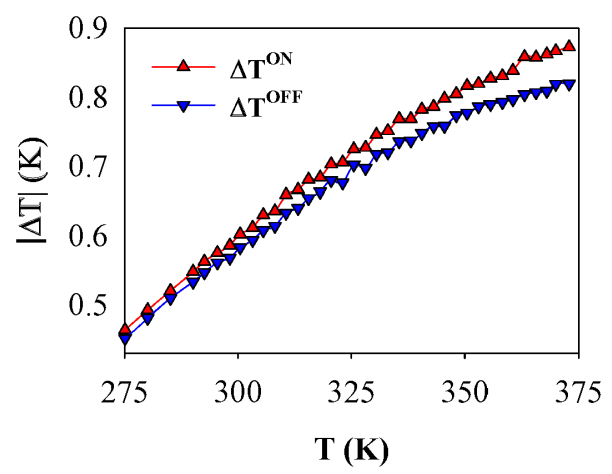

Fig. 10. Temperature dependences of the intensive ECE in applying $|\Delta T|^{\mathrm{ON}}$ and removal $|\Delta T|^{\mathrm{OFF}}$ modes at $E=308 \mathrm{kV} / \mathrm{cm}$. 


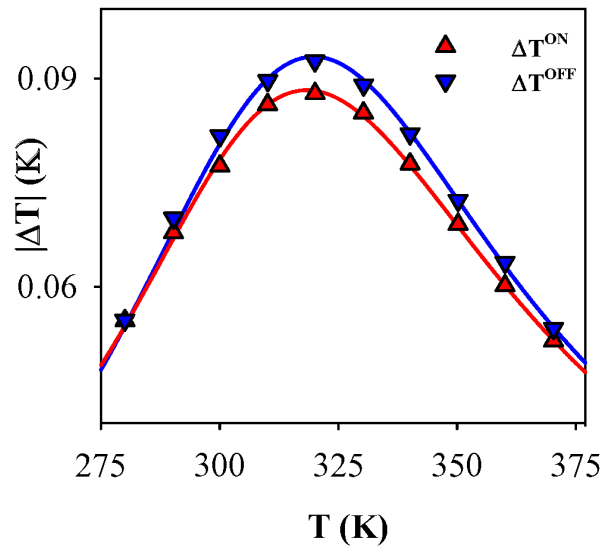

Fig. 11. Temperature dependence of ECE under $E=15.4 \mathrm{kV} / \mathrm{cm}$ at $(d E / d t)^{\mathrm{OFF}}=(d E / d t)^{\mathrm{ON}}$.

$E=15.4 \mathrm{kV} / \mathrm{cm}$, which exceeds the error in determining of the intensive ECE. Thus, the amount of absorbed heat exceeds the heat released. This means that the hypothesis of the possibility of cooling the EC element in a periodic electric field under the condition $\Delta T^{\mathrm{ON}}<\Delta T^{\mathrm{OFF}}$ is realistic.

At the next stage, we performed the investigation of ECE under a periodic field of a different frequency. Measurements were carried out at $T_{\text {bot }}=310 \mathrm{~K}=$ const close to the temperature of the maximum of $(\partial P / \partial T)_{E}$ under $E=15.4 \mathrm{kV} / \mathrm{cm}$. Figures 12(a) and 12(b) show the results of measurements at two frequencies $f=0.025 \mathrm{~Hz}$ and $0.016 \mathrm{~Hz}$, respectively. One can see that the steady state of MLCBT associated with the oscillations of $T_{\text {top }}$ near some average value $\left[\left(\Delta T^{\mathrm{ON}}+\Delta T^{\mathrm{OFF}}\right) / 2\right]_{\max }$ was achieved after several pulses of $E$ (Fig. 12).

Influence of the frequency of the electric field on cooling effect is rather strong. Indeed, the decrease in $f$ from 0.025 to $0.016 \mathrm{~Hz}$ is accompanied by the following points: (1) the values of $\left|\Delta T^{\mathrm{ON}}\right|$ and $\left|\Delta T^{\mathrm{OFF}}\right|$ decrease and increase from 0.040 and $0.078 \mathrm{~K}$ to 0.026 and $0.089 \mathrm{~K}$, respectively; (2) the value $\left[\left(\Delta T^{\mathrm{ON}}+\Delta T^{\mathrm{OFF}}\right) / 2\right]_{\max }$ drops from $-0.019 \mathrm{~K}$ $-0.032 \mathrm{~K}$.

We would like to note, that indeed the value of the cooling is not as great as expected. The observed insignificant value of $\left[\left(\Delta T^{\mathrm{ON}}+\Delta T^{\mathrm{OFF}}\right) / 2\right]_{\max }$ in the periodic electric field may be due to the following circumstances: EC element based on doped $\mathrm{BaTiO}_{3}$ has significant dielectric losses; its dielectric properties strongly depend on the applied electric field; an internal electric field has an inhomogeneous distribution at the ends of the interdigitated electrodes which leads to the appearance of a strong depolarizing field in the multilayer structure.

However, obtained cooling value is an order of magnitude larger than the value presented in Refs. 18 and 19 and suggested cooling method can be amplified by the cascading inclusion of electrocaloric elements without additional thermal switches.

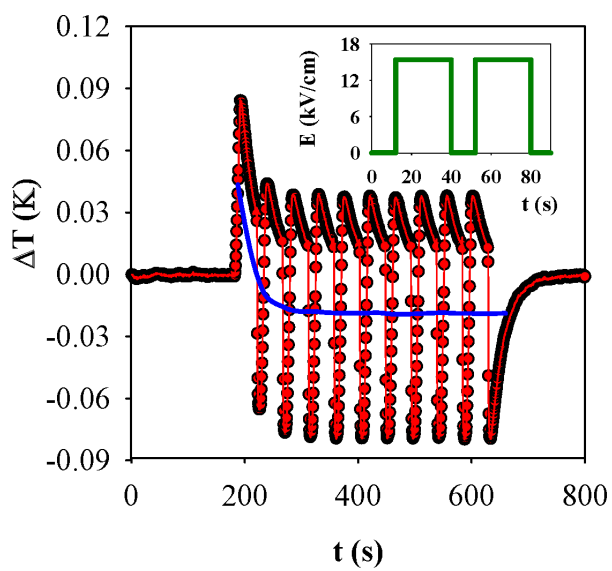

(a)

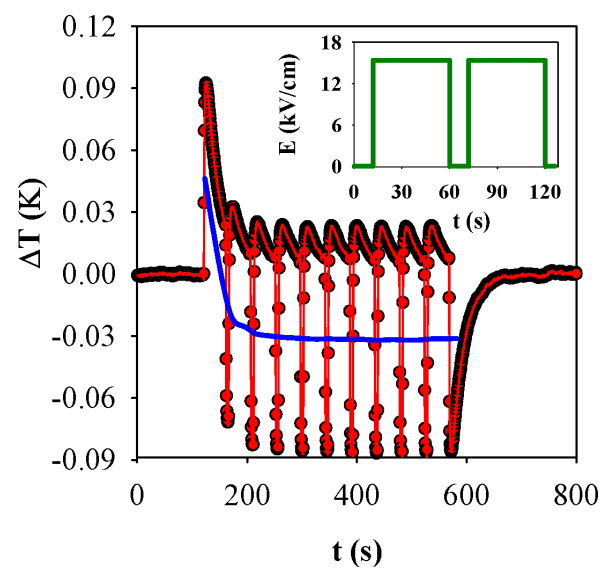

(b)

Fig. 12. Time dependence of the temperature difference $\Delta T=$ $T_{\text {top }}-T_{\text {bot }}$ on the multilayer condenser in a periodic electric field of $E=15.4 \mathrm{kV} / \mathrm{cm}$ at $310 \mathrm{~K}$ : (a) $f=0.025$ and (b) $0.016 \mathrm{~Hz}$. Solid lines show the difference between the $T_{\text {top }}$ and constant $T_{\text {bot }}$ values. Insets: electric field profile.

In order to increase the observed effect, it is necessary to improve the quality of the EC element in order to increase the susceptibility to high periodic electric fields, to reduce the dielectric losses and to decrease the time processes of depolarization/polarization.

\section{Conclusions}

We performed research of thermal expansion, heat capacity, permittivity and polarization of commercial multilayer capacitor based on doped $\mathrm{BaTiO}_{3}$ (MLCBT). One broad anomaly typical for relaxor materials was found in the behavior of these physical properties. In accordance with this, the intensive ECE, $\Delta T$, studied under equilibrium and nonequilibrium thermal conditions also shows one anomaly smeared in a wide temperature range. A high reversibility of 
ECE and good agreement between the values of $\Delta T_{\mathrm{AD}}$ determined under equilibrium thermal conditions by direct (at $S=$ const) and indirect measurements at the electric field $E=15.4 \mathrm{kV} / \mathrm{cm}: \Delta T_{\mathrm{AD}}^{\mathrm{MAX}}=0.104 \mathrm{~K}$ and $\left(\Delta T_{\mathrm{AD}}^{\mathrm{calc}}\right)^{\mathrm{MAX}}=$ $0.107 \mathrm{~K}$ were found. An increase in $E$ to $308 \mathrm{kV} / \mathrm{cm}$ leads to strong increase of ECE to a value of $\Delta T_{\mathrm{AD}}^{\mathrm{MAX}}=0.96 \mathrm{~K}$ which exceeds $\Delta T=0.55 \mathrm{~K}$ determined for related $\mathrm{BaTiO}_{3}$ capacitor at the same $E$ but under nonadiabatic conditions. ${ }^{12}$

The study of the influence of nonequilibrium (quasi-isothermal) conditions on ECE revealed a great role of the relation between the rates of applying, $(d E / d t)^{\mathrm{ON}}$, and removal, $(d E / d t)^{\mathrm{OFF}}$, of the electric field. It was found that the conditions $|\Delta T|^{\mathrm{ON}}<|\Delta T|^{\mathrm{OFF}}$, when the amount of absorbed heat exceeds the heat released, can be realized only in the case $(d E / d t)^{\mathrm{ON}}=(d E / d t)^{\mathrm{OFF}}$. Measurements of $|\Delta T|^{\mathrm{ON}}$ and $|\Delta T|^{\mathrm{OFF}}$ under periodic $E$ have shown that the temperature of the "free" top, $T_{\text {top }}$, decreases in comparison with the temperature of the bottom, $T_{\text {bot }}=$ const. The difference $\left|T_{\text {top }}-T_{\text {bot }}\right|$ was found strongly dependent on the frequency of $E$.

Thus, a commercially available multilayer capacitor based on ferroelectrics can be used as the electrocaloric element which makes the practical application of microrefrigerator without thermal switches. MLCBT has the ideal geometry for the EC element because of the possibility of combination of both high breakdown field and large thermal mass.

\section{Acknowledgments}

The reported study was funded by Russian Foundation for Basic Research, Government of Krasnoyarsk Territory, Krasnoyarsk Region Science and Technology Support Fund to the research, project no. 16-42-240428 r_a, and partially financially supported by Government of Russian Federation, Grant 074-U01.

\section{References}

${ }^{1}$ J. F. Scott, Electrocaloric materials, Ann. Rev. Mat. Res 41, 229 (2011).

${ }^{2} \mathrm{M}$. Valant, Electrocaloric materials for future solid-state refrigeration technologies, Prog. Mater. Sci. 57, 980 (2012).

${ }^{3}$ L. Mañosa, A. Planes and M. Acet, Advanced materials for solidstate refrigeration, J. Mater. Chem. A 1, 4925 (2013).

${ }^{4}$ X. Moya, S. Kar-Narayan and N. D. Mathur, Caloric materials near ferroic phase transitions, Nat. Mater. 13, 439 (2014).

${ }^{5}$ L. Mañosa and A. Planes, Materials with giant mechanocaloric effects: Cooling by strength, Adv. Mater. 29, 1603607 (2017).

${ }^{6}$ E. A. Mikhaleva, I. N. Flerov, A. V. Kartashev, M. V. Gorev, E. V. Bogdanov and V. S. Bondarev, Thermal, dielectric and barocaloric properties of $\mathrm{NH}_{4} \mathrm{HSO}_{4}$ crystallized from an aqueous solution and the melt, Solid State Sci. 67, 1 (2017).

${ }^{7}$ I. Ponomareva and S Lisenkov, Bridging the Macroscopic and Atomistic Descriptions of the Electrocaloric Effect, Phys. Rev. Lett. 108, 167604 (2012).
${ }^{8}$ G. C. Lin, X. M. Xiong, J. X. Zhang and Q. Wei, Latent heat study of phase transition in $\mathrm{Ba}_{0.73} \mathrm{Sr}_{0.27} \mathrm{TiO}_{3}$ induced by electric field, J. Therm. Anal. Calorim. 81, 41 (2005).

${ }^{9}$ H. Y. Lee, K. H. Cho and H.-D. Nam, Grain Size and Temperature Dependence of Electrical Breakdown in $\mathrm{BaTiO}_{3}$ Ceramic, Ferroelectrics 334, 165 (2006).

${ }^{10}$ A. S. Mischenko, Q. M. Zhang, J. F. Scott, R. W. Whatmore and N. D. Mathur, Giant electrocaloric effect in thin-film $\mathrm{PbZr}_{0.95} \mathrm{Ti}_{0.0503}$, Science 311, 270 (2006).

${ }^{11}$ R. I. Epstein and K. J. Malloy, Electrocaloric devices based on thin-film switches, J. Appl. Phys. 106, 064509 (2009).

${ }^{12}$ S. Kar-Narayan and N. D. Mathur, Direct and indirect electrocaloric measurements using multilayer capacitors, J. Phys. D, Appl. Phys. 43, 032002 (2010).

${ }^{13}$ S. J. Smullin, Y. Wang and D. E. Schwartz, System optimization of a heat-switch-based electrocaloric heat pump, Appl. Phys. Lett. 107, 093903 (2015).

${ }^{14}$ Y. Wang, D. E. Schwartz, S. J. Smullin, Q. Wang and M. J. Sheridan, Silicon Heat Switches for Electrocaloric Cooling, J. Microelectromech. Syst. 26, 580 (2017).

${ }^{15}$ S. F. Karmanenko, O. V. Pakhomov, A. M. Prudan, A. S. Starkov and A. V. Es'kov, Layered ceramic structure based on the electrocaloric elements working as a solid-state cooling line, J. Eur. Ceram. Soc. 27, 3109 (2007).

${ }^{16}$ A. V. Es'kov, S. F. Karmanenko, O. V. Pakhomov and A. S. Starkov, Simulation of a solid-state cooler with electrocaloric elements, Phys. Solid State 51, 1574 (2009).

${ }^{17}$ A. S. Starkov, S. F. Karmanenko, O. V. Pakhomov, A. V. Es'kov, D. Semikin and J. Hagberg, Electrocaloric response of a ferroelectric capacitor to a periodic electric field, Phys. Solid State 51, 1510 (2009).

${ }^{18}$ V. S. Bondarev, E. A. Mikhaleva, M. V. Gorev and I. N. Flerov, Intensive electrocaloric effect in triglicine sulfat under nonequilibrium thermal conditions and periodic electric field, Phys. Stat. Sol. B 253, 2073 (2016).

${ }^{19}$ V. S. Bondarev, E. A. Mikhaleva, I. N. Flerov and M. V. Gorev, Electrocaloric effect in triglicine sulfat under equlibrium and nonequilibrium thermodynamic conditions, Phys. Solid State 59, 1118 (2017).

${ }^{20}$ B. Kwon, I. J. Roh, S. H. Baek, S. K. Kim, J. S. Kim and C. Y. Kang, Dynamic temperature response of electrocaloric multilayer capacitors, Appl. Phys. Lett. 104, 213902 (2014).

${ }^{21}$ Bruker AXS TOPAS V4: General profile and structure analysis software for powder diffraction data. User's Manual (Bruker AXS, Karlsruhe, Germany. 2008).

${ }^{22}$ A. V. Kartashev, I. N. Flerov, N. V. Volkov and K. A. Sablina, Adiabatic calorimetric study of the intense magnetocaloric effect and the heat capacity of $\left(\mathrm{La}_{0.4} \mathrm{Eu}_{0.6}\right)_{0.7} \mathrm{~Pb}_{0.3} \mathrm{MnO}_{3}$, Phys. Solid State 50, 2115 (2008).

${ }^{23}$ A. V. Kartashev, E. A. Mikhaleva, M. V. Gorev, E. V. Bogdanov, A. V. Cherepakhin, K. A. Sablina and N. V. Volkov, Thermal properties, magneto- and baro-caloric effects in $\mathrm{La}_{0.7} \mathrm{~Pb}_{0.3} \mathrm{MnO}_{3}$ single crystal, J. Appl. Phys. 113, 73901 (2013).

${ }^{24}$ H. Shin, J. S. Park, K. S. Hong, H. S. Jung, J. K. Lee and K. Y. Rhee, Physical origin of residual thermal stresses in a multilayer ceramic capacitor, J. Appl. Phys. 101, 063527 (2007).

${ }^{25}$ J. S. Park, S. Kim, H. Shin, H. S. Jung and K. S. Hong, Residual stress evolution in multilayer ceramic capacitors corresponding to layer increase and its correlation to the dielectric constant, J. Appl. Phys. 97, 094504 (2005). 
${ }^{26}$ Y. Gang, Y. Zhenxing, S. Tieyu, G. Huanlin and L. Longtu, Effect of combined external uniaxial stress and dc bias on the dielectric property of $\mathrm{BaTiO}_{3}$-based dielectrics in multilayer ceramic capacitor: Thermodynamics and experiments, J. Phys. D, Appl. Phys. 41, 45307 (2008).

${ }^{27}$ C. Lei, A. A. Bokov and Z.-G. Ye, Ferroelectric to relaxor crossover and dielectric phase diagram in the $\mathrm{BaTiO}_{3}-\mathrm{BaSnO}_{3}$ system, J. Appl. Phys. 101, 084105 (2007).

${ }^{28}$ W. J. Merz, Double Hysteresis Loop of $\mathrm{BaTiO}_{3}$ at the Curie Point, Phys. Rev. 91, 513 (1953).

${ }^{29}$ S. K. Upadhyay, V. R. Reddy, P. Bag, R. Rawat, S. M. Gupta and A. Gupta, Electro-caloric effect in lead-free $\mathrm{Sn}$ doped $\mathrm{BaTiO}_{3}$ ceramics at room temperature and low applied fields, Appl. Phys. Lett. 105, 112907 (2014).

${ }^{30}$ W. Cai, Y. Fan, J. Gao, Ch. Fu and X. Deng, J. Mater. Sci., Mater. Electron. 22, 265 (2011).

${ }^{31}$ L. Xie, Y. L. Li, R. Yu, Z. Y. Cheng, X. Y. Wei, X. Yao, C. L. Jia, K. Urban, A. A. Bokov, Z.-G. Ye and J. Zhu, Static and dynamic polar nanoregions in relaxor ferroelectric $\mathrm{Ba}\left(\mathrm{Ti}_{1-x} \mathrm{Sn}_{x}\right) \mathrm{O}_{3}$ system at high temperature, Phys. Rev. B 85, 014118 (2012).

${ }^{32}$ V. V. Shvartsman, W. Kleemann, J. Dec, Z. K. Xu and S. G. Lu, Diffuse phase transition in $\mathrm{BaTi}_{1-x} \mathrm{Sn}_{x} \mathrm{O}_{3}$ ceramics: An intermediate state between ferroelectric and relaxor behavior, J. Appl. Phys. 99, 124111 (2006). 